\title{
EFEITO DE INDUTORES DE FLORESCIMENTO NAS CULTIVARES DE ABACAXIZEIRO RBR-1, SNG-2 e SNG-3 EM RIO BRANCO-ACRE ${ }^{1}$
}

\author{
ANA DA SILVA LEDO ${ }^{2}$, TARCÍSIO MARCOS DE SOUZA GONDIM ${ }^{3}$, TADÁRIO KAMEL DE OLIVEIRA4 \\ JACSON RONDINELLI DA SILVA NEGREIROS ${ }^{5}$, FRANCISCO FELISMINO DE AZEVEDO ${ }^{6}$
}

\begin{abstract}
RESUMO - Este trabalho teve como objetivo avaliar o efeito de indutores de florescimento em três cultivares de abacaxizeiro em Rio Branco-Acre. O delineamento experimental foi em blocos ao acaso, em esquema fatorial 3 × 2 x 2, com quatro repetições, sendo combinadas três cultivares de abacaxi (RBR-1, SNG-2 e SNG-3), dois indutores de florescimento (carbureto de cálcio- $\mathrm{CaC}_{2}$ e etefon-ácido 2-cloroetilfosfônico) e duas épocas de aplicação (aos 10 e 12 meses do plantio). As plantas induzidas com etefon aos 10 meses apresentaram maior percentagem de florescimento (96,25\%) quando comparadas com as induzidas com $\mathrm{CaC}_{2}(62,92 \%)$. A percentagem de florescimento foi significativamente superior nas plantas induzidas aos 12 meses com $\mathrm{CaC}_{2}(85,42 \%)$ quando comparadas com as induzidas aos 10 meses $(62,92 \%)$.
\end{abstract}

Termos para indexação: Ananas comosus, regulador de crescimento, ciclo de produção.

\section{FLORAL DIFFERENTIATION OF RBR-1, SNG-2 AND SNG-3 PINEAPPLE CULTIVARS IN RIO BRANCO-ACRE,} BRAZIL

ABSTRACT- The aim of this study was to evaluate the influence of floral inductors in the floral differentiation of pineapple in the conditions of Rio Branco, AC, Brazil. The experimental design was a complete randomized block in factorial $3 \times 2 \times 2$, with four replications, three pineapple cultivars combined (RBR-1, SNG-2 and SNG-3), two floral inductors (calcium carbide- $\mathrm{CaC}_{2}$ and (2 chloroethyl) phosphonic acid -ethefon) and two application times (10 and 12 months after planting). The plants induced with ethefon after 10 months presented larger floral differentiation (96.25\%) when compared with $\mathrm{CaC}_{2}(62.92 \%)$. The floral differentiation was superior significantly in the plants induced after 12 months with $\mathrm{CaC}_{2}(85.42 \%)$ when compared with 10 months $(62.92 \%)$.

Index Terms: Ananas comosus, growth regulator, production cycle.

\section{INTRODUÇÃO}

No Brasil, foram colhidos, no ano de 2001, 62.597 ha de abacaxizeiro (Ananas comosus (L.) Merril) com valor da produção estimado em 690,36 mil reais, destacando-se os Estados de Minas Gerais, Paraíba e Pará (IBGE, 2003). No Estado do Acre, a área cultivada tem aumentado nos últimos anos com a utilização de cultivares locais selecionadas e recomendadas pela Embrapa Acre: RBR-2 'Cabeça-deonça', SNG-2 ‘Quinari', RBR-1 'Rio Branco' e 'SNG-3'. As cultivares apresentam padrão semelhante ao do grupo 'Cayenne' e floração natural, aproximadamente, entre os 15 e 18 meses após o plantio (Ritzinger, 1992).

Segundo Sampaio et al. (1997), a diferenciação floral natural pode contribuir para a diminuição do rendimento quando ocorre de maneira desuniforme, pois determina variações na época de maturação dos frutos, dificulta os tratos fitossanitários e a colheita. O abacaxizeiro responde muito bem à aplicação de substâncias químicas que apresentem a capacidade de influenciar alguns de seus processos fisiológicos, especialmente o florescimento, que permite o seu cultivo comercial de forma racional e econômica (Cunha, 1999). Existem poucas informações técnicas à disposição dos produtores sobre a indução floral do abacaxizeiro para escalonamento da produção nas condições climáticas do Acre. O florescimento natural das cultivares ocorre tardiamente, prolongando o ciclo de produção. Conforme Ritzinger (1991), a cultivar SNG-3 inicia o florescimento aos 15 meses do plantio, e o período compreendido entre o surgimento da inflorescência e a colheita do fruto é de 118 dias, apresentando ciclo de produção longo, em torno de 568 dias.

A indução artificial do florescimento do abacaxizeiro, dentre diversas vantagens, reduz os efeitos negativos da floração natural, altamente heterogênea e antecipa a produção (Cunha et al., 1993).

Várias substâncias podem ser utilizadas, como o carbureto de cálcio e o ácido 2-cloroetilfosfônico (etefon). Estas substâncias diferem quanto ao modo de aplicação, sendo que o carbureto de cálcio pode ser aplicado sob a forma sólida na dosagem de 05 a 1,0 g/planta, em períodos chuvosos, ou na forma líquida 30 a $50 \mathrm{~mL} /$ planta, em épocas frias (Cunha, 1999). A dose geralmente recomendada para o etefon é de 1 a 4 litros do produto comercial/1000 L de água. Entretanto a diminuição da acidez da solução indutora eleva significativamente sua eficiência, possibilitando o uso de menor quantidade do produto (Cunha, 1999).

Diversos trabalhos têm sido conduzidos para avaliar a melhor época para a indução floral em diferentes regiões do País (Bezerra et al., 1978; Choairy \& Fernandes, 1984; Choairy et al., 1994a, 1994b). Em estudos conduzidos por Gondim \& Azevedo (2002) em Rio Branco, Acre, o carbureto de cálcio promoveu a floração da cv. SNG-3, apresentando maior eficiência quando aplicado aos 10 meses do plantio.

Este trabalho teve como objetivo avaliar o efeito da aplicação do carbureto de cálcio e de etefon aos 10 e 12 meses após o plantio, em três cultivares de abacaxizeiro, nas condições edafoclimáticas de Rio Branco, Acre.

\section{MATERIAL E MÉTODOS}

Este trabalho foi conduzido no Campo Experimental da Embrapa Acre, em Rio Branco-AC, com coordenadas de S 1001'30"e W 67²4' $18^{\prime}$ ', e altitude de aproximadamente $160 \mathrm{~m}$. Utilizaram-se as cultivares de abacaxi RBR-1, SNG-2 e SNG-3, que apresentam padrão semelhante ao da cv. Smooth Cayenne (Ritzinger, 1991). O clima é quente e úmido, com estações seca e úmida bem definidas. O solo é classificado como Argissolo Vermelho de topografia plana, apresentando as seguintes características químicas: $\mathrm{pH}$ (água) $=5,0 ; \mathrm{P}=1,0 \mathrm{mg} \mathrm{dm}^{-3} ; \mathrm{K}=0,10 \mathrm{cmol}_{\mathrm{c}} \mathrm{dm}^{-3} ; \mathrm{Ca}+\mathrm{Mg}$ $=3,20 \mathrm{cmol}_{\mathrm{c}} \mathrm{dm}^{-3} ; \mathrm{Al}=0,20 \mathrm{cmol}_{\mathrm{c}} \mathrm{kg}^{-1}, \mathrm{e} \mathrm{C}=0,53 \mathrm{~g} \mathrm{~kg}^{-1}$.

O plantio foi realizado no dia 21 de janeiro de 2001, utilizando

\footnotetext{
1 (Trabalho 127/2003). Recebido: 11/09/2003. Aceito para publicação: 20/08/2004.

${ }^{2}$ Eng. Agra. D.Sc., Pesquisadora Embrapa Tabuleiros Costeiros, C.P.44, CEP 49025-040 Aracaju-SE. Tel. 79-2261318. analedo@cpatc.embrapa.br

${ }^{3}$ Eng. Agr., M.Sc., Pesquisador Embrapa Algodão, C.P.174, CEP 58107-720, Campina Grande, PB. Tel.:83-315-4300.

tarcisio@cnpa.embrapa.br.

${ }^{4}$ Eng. Agr. M.Sc. Pesquisador Embrapa Acre, C.P.321, CEP 69908-970, Rio Branco, AC, Tel. 79-2243200, tadario@cpafac.embrapa.br

${ }^{5}$ Eng. Agr. Mestrando UFV, R. Juquinha de Paula nº 56/201, B.Santo Antônio, CEP: 36570-000 - Viçosa -MG.

jacson@viçosa.ufv.br

${ }^{6}$ Eng. Agr., Assistente de Pesquisa da Embrapa Acre, 79-2243200, felizmino@ cpafac.embrapa.br
} 
TABELA 1 - Resumo da análise de variância da percentagem de florescimento, do número de filhotes e número de rebentões em três cultivares de abacaxizeiro, em função da idade das plantas e do indutor de florescimento. Rio Branco-AC, 2003.

\begin{tabular}{|c|c|c|c|c|}
\hline \multirow[b]{2}{*}{ Fonte de Variação } & \multirow[b]{2}{*}{ GL } & \multicolumn{3}{|c|}{ Quadrados Médios } \\
\hline & & $\begin{array}{l}\text { Percentagem de } \\
\text { florescimento }\end{array}$ & Número de filhotes & Número de rebentões \\
\hline Repetição & 3 & $428,29 \mathrm{~ns}$ & $2,76 \mathrm{~ns}$ & $0,011 \mathrm{~ns}$ \\
\hline Cultivar (C) & 2 & $1073,44 * *$ & $36,14 * *$ & $0,094 * *$ \\
\hline Indutor (I) & 1 & $1575,52 * *$ & $3,56 \mathrm{~ns}$ & $0,042 \mathrm{~ns}$ \\
\hline Idade (P) & 1 & $5963,02 * *$ & $128,28 * *$ & $0,258 * *$ \\
\hline C X I & 2 & $78,64 n s$ & $0,75 \mathrm{~ns}$ & $0,274 \mathrm{~ns}$ \\
\hline C X P & 2 & $725,52 \mathrm{~ns}$ & $18,27 * *$ & $0,132 * *$ \\
\hline I X P & 1 & $1463,02 * *$ & 4,38ns & $0,017 \mathrm{~ns}$ \\
\hline C X I X P & 2 & $691,15 \mathrm{~ns}$ & $0,85 \mathrm{~ns}$ & $0,006 \mathrm{~ns}$ \\
\hline Erro & 33 & 266,55 & 2,40 & 0,017 \\
\hline CV (\%) & & 19,14 & 26,48 & 14,55 \\
\hline
\end{tabular}

1 - Variável transformada em raiz quadrada de $\mathrm{Y}+0,5$

** - Significativo a $1 \%$ de probabilidade, pelo teste $\mathrm{F}$.

ns - Não significativo.

mudas tipo filhote, com peso variando de 250 a 350 gramas, em covas no espaçamento $0,90 \mathrm{~m} \times 0,40 \mathrm{~m}$, em fileiras simples. Os tratos culturais e adubações seguiram as recomendações de Reinhardt et al. (2000). O delineamento experimental foi em blocos ao acaso, com quatro repetições, em esquema fatorial, sendo combinados três cultivares de abacaxi (RBR1, SNG-2 e SNG-3), dois indutores de florescimento (carbureto de cálcio$\mathrm{CaC}_{2}$ e ácido 2-cloroetilfosfônico-etefon) e duas épocas de aplicação (aos 10 e 12 meses do plantio), totalizando doze tratamentos. As parcelas foram constituídas por 48 plantas distribuídas em quatro filas de 12 plantas, sendo a parcela útil composta de 20 plantas das duas filas centrais.

Foram aplicados dois indutores florais às $07 \mathrm{~h} 30 \pm 20$ ' da manhã: o carbureto de cálcio comercial, na forma sólida, em pedras com peso médio de $0,8 \mathrm{~g} /$ planta em única aplicação na roseta foliar, com auxílio de um funil de tubo prolongado e o etefon na concentração de 2 litros do produto comercial/1000 L de água/ha com a adição de hidróxido de cálcio (35g/100 L da solução), aplicando-se $30 \mathrm{~mL}$ da solução na planta (Cunha , 1999).

Avaliaram-se os seguintes caracteres: dias do plantio até a colheita; percentagem de floração até os 60 dias após o tratamento de indução; número de filhotes; número de rebentos (mudas tipo filhoterebentão e rebentão); peso do fruto com e sem coroa; peso da coroa; acidez total titulável do suco-ATT (\% de ácido cítrico); sólidos solúveis totais (SST), por meio de refratômetro manual, com posterior correção dos valores observados para temperatura ambiente, de acordo com Pregnolatto \& Pregnolatto (1985), e relação SST/ATT. As médias foram submetidas à análise de variância e, quando significativas, comparadas pelo teste de Tukey, a 5\% de probabilidade.

\section{RESULTADOS E DISCUSSÃO}

Houve diferenças significativas na percentagem de florescimento (PF), número de filhotes (NF), número de rebentões (NR), peso do fruto com coroa (PFCC) e sem coroa (PFSC), peso da coroa (PC), sólidos solúveis totais (SST), acidez titulável total (ATT) e relação SST/ ATT para as cultivares e idade da planta (Tabelas 1 e 2). Para todas as variáveis analisadas, com exceção da percentagem de florescimento e relação SST/ATT, não houve diferenças significativas para o tipo de indutor de florescimento (Tabelas 1 e 2). Houve efeitos significativos da interação cultivar x idade da planta $(\mathrm{C} \times \mathrm{P})$ para número de filhotes e de rebentos e da interação tipo de indutor de florescimento $\mathrm{x}$ idade da planta (I x P) para percentagem de florescimento e peso da coroa (Tabelas 1 e 2).

As cultivares induzidas aos 10 e 12 meses apresentaram um ciclo de produção de 475 e 558 dias, respectivamente, sendo que as parcelas que não receberam tratamento de indução artificial iniciaram o florescimento por ocasião da colheita das plantas induzidas aos 12 meses de idade.

Apesar de não ter sido detectada interação significativa entre as cultivares e os demais fatores estudados, em média, todas as cultivares induzidas com etefon aos 10 meses apresentaram maior percentagem de florescimento $(96,25 \%)$ quando comparadas com as induzidas com $\mathrm{CaC}_{2}$ $(62,92 \%)$. Não foram verificadas diferenças significativas entre os indutores aplicados aos 12 meses quanto à percentagem de florescimento (Tabela 3). Entretanto a percentagem de florescimento foi significativamente superior nas plantas induzidas aos 12 meses com $\mathrm{CaC}_{2}(85,42 \%)$ quando comparadas com as induzidas aos 10 meses de

TABELA 2 - Resumo da análise de variância do peso do fruto com e sem coroa, peso da coroa, acidez titulável total (ATT), sólidos solúveis totais em \% de ácido cítrico (SST) e relação SST/ATT, em três cultivares de abacaxizeiro, em função da idade da planta (P) e do indutor de florescimento (I). Rio Branco-AC, 2003.

\begin{tabular}{|c|c|c|c|c|c|c|c|}
\hline \multirow[b]{2}{*}{$\begin{array}{l}\text { Fonte } \\
\text { de Variação }\end{array}$} & \multirow[b]{2}{*}{$\mathrm{GL}$} & \multicolumn{6}{|c|}{ Quadrados Médios } \\
\hline & & $\begin{array}{l}\text { Peso do fruto com } \\
\text { coroa }(\mathrm{g})\end{array}$ & $\begin{array}{l}\text { Peso do fruto sem } \\
\text { coroa }(\mathrm{g})\end{array}$ & $\begin{array}{c}\text { Peso da } \\
\text { coroa }(\mathrm{g})\end{array}$ & SST & ATT & $\begin{array}{l}\mathrm{SST} / \\
\mathrm{ATT}\end{array}$ \\
\hline Repetição & 3 & $75682,28 \mathrm{~ns}$ & $57311,90 \mathrm{~ns}$ & $3441,88^{*}$ & $0,6151 \mathrm{~ns}$ & 0,0030ns & $2,49 \mathrm{~ns}$ \\
\hline Cultivar (C) & 2 & $492833,66^{* *}$ & $802940,48^{* *}$ & $37973,20 * *$ & $1,8015 \mathrm{~ns}$ & $0,0549 *$ & $169,67 *$ \\
\hline Indutor (I) & 1 & $48757,27 \mathrm{~ns}$ & $57437,38 \mathrm{~ns}$ & $355,34 \mathrm{~ns}$ & $0,1668 \mathrm{~ns}$ & $0,040 \mathrm{~ns}$ & $136,72 *$ \\
\hline Idade (P) & 1 & $897977,11 * *$ & $1053352,43 * *$ & $6195,74^{*}$ & $0,046 \mathrm{~ns}$ & $0,2898 * *$ & $531,67 * *$ \\
\hline C X I & 2 & $128300,92 \mathrm{~ns}$ & $116181,60 \mathrm{~ns}$ & $1783,86 \mathrm{~ns}$ & $0,2828 \mathrm{~ns}$ & $0,0330 \mathrm{~ns}$ & $77,04 \mathrm{~ns}$ \\
\hline C X P & 2 & $44052,10 \mathrm{~ns}$ & $45893,20 \mathrm{~ns}$ & $3161,87 \mathrm{~ns}$ & $0,1214 \mathrm{~ns}$ & $0,0106 \mathrm{~ns}$ & $2,56 \mathrm{~ns}$ \\
\hline I X P & 1 & $43643,93 n s$ & $15746,28 \mathrm{~ns}$ & $6960,08 *$ & $2,6273 \mathrm{~ns}$ & $0,0111 \mathrm{~ns}$ & $2,54 \mathrm{~ns}$ \\
\hline C X I X P & 2 & $14432,80 \mathrm{~ns}$ & $9300,43 \mathrm{~ns}$ & $594,51 \mathrm{~ns}$ & $0,2562 \mathrm{~ns}$ & $0,0010 \mathrm{~ns}$ & $1,02 \mathrm{~ns}$ \\
\hline Erro & 33 & 72715,79 & 71395,78 & 1157,67 & $0,9737 \mathrm{~ns}$ & 0,0148 & 39,15 \\
\hline CV $(\%)$ & & 18,52 & 20,73 & 20,32 & 7,10 & 21,20 & 24,25 \\
\hline
\end{tabular}

*, ** - Significativo a $5 \%$ e a $1 \%$ de probabilidade, respectivamente, pelo teste $\mathrm{F}$.

ns - Não significativo. 
TABELA 3 - Médias da percentagem de florescimento, número de fillhotes e número de rebentões de três cultivares de abacaxizeiro, em função do indutor de florescimento e da idade das plantas. Rio Branco-AC, 2003.

\begin{tabular}{|c|c|c|c|}
\hline \multicolumn{4}{|c|}{ Percentagem de Florescimento $^{1}$} \\
\hline Idade da planta/Tipo de Indutor & Carbureto de Cálcio & & Etefon \\
\hline 10 meses (475 dias) & $62,92 \mathrm{Bb}$ & & $96,25 \mathrm{Aa}$ \\
\hline 12 meses (558 dias) & $85,42 \mathrm{Aa}$ & & $96,67 \mathrm{Aa}$ \\
\hline Cultivar & RBR-1 & SNG-2 & SNG-3 \\
\hline \multirow{2}{*}{\multicolumn{4}{|c|}{$\frac{87,50 \mathrm{ab}}{\text { Número de filhotes }^{1}}$}} \\
\hline & & & \\
\hline Idade da Planta/Cultivar & RBR-1 & SNG-2 & SNG-3 \\
\hline 10 meses (475 dias) & $3,38 \mathrm{aB}$ & $4,80 \mathrm{aB}$ & $4,49 \mathrm{aA}$ \\
\hline 12 meses (558 dias) & $6,29 \mathrm{bA}$ & $10,36 \mathrm{aA}$ & $5,80 \mathrm{bA}$ \\
\hline \multicolumn{4}{|c|}{ Número de rebentos $^{1}$} \\
\hline Idade da Planta/Cultivar & RBR-1 & SNG-2 & SNG-3 \\
\hline 10 meses (475 dias) & $0,09 \mathrm{bB}$ & $0,00 \mathrm{bB}$ & $0,60 \mathrm{aA}$ \\
\hline 12 meses (558 dias) & $0,45 \mathrm{aA}$ & $0,51 \mathrm{aA}$ & $0,44 \mathrm{aA}$ \\
\hline
\end{tabular}

${ }^{1}$ Médias seguidas da mesma letra minúscula na linha e maiúscula na coluna, dentro de cada fator ou interação na coluna, não diferem entre si, a 5\% de probabilidade, pelo teste de Tukey.

idade (62,92\%). Gondim \& Azevedo (2002) verificaram também maior florescimento em plantas induzidas com $\mathrm{CaC}_{2}$ aos 12 meses de idade. Não houve diferenças significativas na indução do florescimento com etefon, nas duas idades de aplicação. A resposta ao tratamento de indução artificial varia de acordo com o vigor e taxa de crescimento da planta (Cunha, 1999). As cultivares RBR-1, SNG-2 e SNG-3 apresentaram, em média, 76,25; 87,50 e 92,18\% de florescimento, respectivamente (Tabela 3), concordando com relatos de Cunha et al. (1999), de que nem todas as variedades respondem igualmente aos estímulos florais.

As cultivares RBR-1 e SNG-2 apresentaram maior número de mudas tipo filhote e número de rebentos por ocasião da colheita quando induzidas aos 12 meses de idade. Resultados semelhantes foram obtidos por Choairy et al. (1994a) para a cv. Smooth Cayenne com a indução floral realizada aos 12 meses do plantio. Este comportamento pode ser atribuído à maior idade e desenvolvimento vegetativo das plantas aos 12 meses. A cv. SNG-3 não foi influenciada pela idade da indução nas duas variáveis, concordando com os resultados obtidos por Gondim et. al. (2002) para a mesma cultivar quanto à produção de mudas do tipo rebentão.

O tipo de indutor não influenciou nas variáveis obtidas por ocasião da colheita. Entretanto, observou-se que as cultivares induzidas aos 12 meses de idade apresentaram maiores PFCC, PFSC, PC e ATT, promovendo um aumento de 17,2 e $20,6 \%$ no PFCC e PFSC, respectivamente (Tabela 4). Diversos autores alcançaram resultados semelhantes em plantas induzidas mais tardiamente (Vieira et al., 1983; Choairy \& Fernandes, 1984; Cunha et al., 1993; Melo, 1993; Choairy et al., 1994a, 1994b; Gondim \& Azevedo, 2002). O peso do fruto do abacaxizeiro depende, entre outros fatores, especialmente do estado nutricional e estádio de crescimento alcançado pela planta quando da diferenciação floral (Cunha et al., 1999).

Os frutos alcançaram, em média, o PFCC de $1.512,19 \mathrm{~g}$ e o PFSC de 1.437,13 g com a indução aos 12 meses de idade e o PFCC de 1.319,63 g e PFSC de 1.140,85 g com a indução aos 10 meses de idade (Tabela 4), apresentando excelente padrão para comercialização para o consumo in natura e para exportação (Carvalho, 1999). Plantas induzidas com $\mathrm{CaC}_{2}$ e etefon não diferiram estatisticamente entre si e obtiveram, em média, o PFCC de 1.488,28 e PFSC de 1.424,54 g, respectivamente. A cultivar SNG2 obteve, em média, o maior PFSC $(1.527,46 \mathrm{~g})$ e o menor PC $(117,50 \mathrm{~g})$. Observou-se que as plantas induzidas aos 12 meses produziram coroas mais pesadas $(178,79 \mathrm{~g})$ do que aos 10 meses $(156,06 \mathrm{~g})$, discordando dos resultados obtidos por Gondim \& Azevedo (2002) na cv. SNG-3, por Melo (1993) na cv. Pérola e por Manica et al. (1994) na cv. Smooth Cayenne, que obtiveram maiores pesos de coroas nos frutos de plantas induzidas mais precoces (Tabela 4 )

Os frutos não apresentaram diferenças significativas do SST quanto aos fatores estudados, mas as plantas induzidas aos 12 meses de idade produziram frutos com maior acidez e menor relação SST/ATT (Tabela 4). Choary et al. (1994a) observaram que as variáveis SST e ATT em frutos da cv. Smooth Cayenne variaram em função da época de plantio. Gondim \& Azevedo (2002) obtiveram em plantas da cv. SNG-3 induzidas aos 10 e 12 meses de idade frutos com menor acidez e mais ricos em sólidos solúveis totais. Provavelmente, a época de plantio tenha influenciado nas características químicas dos frutos. As modificações metabólicas dos frutos durante a maturação também são afetadas, entre outros fatores, pela cultivar (Carvalho, 1999). Diversos trabalhos sobre a influência da idade de indução floral do abacaxizeiro sobre a qualidade

TABELA 4 - Médias das variáveis peso do fruto com (PFCC) e sem coroa (PFSC), peso da coroa (PC), acidez titulável total (ATT), sólidos solúveis totais (SST) e relação SST/ATT de três cultivares de abacaxizeiro, em função do tipo de indutor de florescimento e idade da planta na aplicação. Rio Branco-AC, 2003.

\begin{tabular}{|c|c|c|c|c|c|c|}
\hline \multirow[b]{2}{*}{ Fatores } & \multicolumn{6}{|c|}{ Médias ${ }^{1}$} \\
\hline & $\operatorname{PFCC}(\mathrm{g})$ & $\operatorname{PFSC}(\mathrm{g})$ & $\mathrm{PC}(\mathrm{g})$ & $\begin{array}{c}\text { SST } \\
\left({ }^{\circ} \text { Brix }\right)\end{array}$ & ATT & $\begin{array}{l}\text { SST/ } \\
\text { ATT }\end{array}$ \\
\hline \multicolumn{7}{|l|}{ Cultivar } \\
\hline RBR-1 & $1.426,49 \mathrm{ab}$ & $1.256,56 \mathrm{~b}$ & $169,92 b$ & $13,79 a$ & $0,55 b$ & $26,93 \mathrm{ab}$ \\
\hline SNG-2 & $1.644,96 \mathrm{a}$ & $1.527,46 \mathrm{a}$ & $117,50 \mathrm{c}$ & $14,27 \mathrm{a}$ & $0,53 b$ & $28,34 a$ \\
\hline SNG-3 & $1.297,80 \mathrm{c}$ & $1.082,96 \mathrm{~b}$ & $214,83 \mathrm{a}$ & $13,62 \mathrm{a}$ & $0,64 \mathrm{a}$ & $22,13 \mathrm{c}$ \\
\hline \multicolumn{7}{|l|}{ Tipo de Indutor } \\
\hline Carbureto de cálcio & $1.488,28 \mathrm{a}$ & $1.323,58 \mathrm{a}$ & $164,70 \mathrm{a}$ & $13,83 \mathrm{a}$ & $0,54 \mathrm{a}$ & $27,49 a$ \\
\hline Etefon & $1.424,54 \mathrm{a}$ & $1.254,40 \mathrm{a}$ & $170,14 \mathrm{a}$ & $13,95 \mathrm{a}$ & $0,60 \mathrm{a}$ & $24,11 \mathrm{a}$ \\
\hline \multicolumn{7}{|l|}{ Idade da Planta } \\
\hline 10 meses (475 dias) & $1.319,63 \mathrm{~b}$ & $1.140,85 b$ & $156,06 b$ & $13,92 \mathrm{a}$ & $0,49 b$ & $29,13 a$ \\
\hline 12 meses (558 dias) & $1.593,19 \mathrm{a}$ & $1.437,13 \mathrm{a}$ & $178,79 a$ & $13,86 \mathrm{a}$ & $0,65 \mathrm{a}$ & $22,47 b$ \\
\hline
\end{tabular}

${ }^{1}$ Médias seguidas da mesma letra, dentro de cada fator na coluna, não diferem entre si, a 5\% de probabilidade, pelo teste de Tukey. 
do fruto também foram conduzidos para a cultivar Smooth Cayenne por Choairy \& Fernandes (1984) e Choairy et al. (1994a) e com a cultivar Pérola (Choairy et al., 1994b).

\section{CONCLUSÕES}

1) Recomenda-se para a indução das cultivares RBR-1, SNG-2 e SNG-3, aos 10 meses de idade, o etefon.

2) Recomenda-se para a indução das cultivares RBR-1, SNG-2 e SNG-3, aos 12 meses de idade, o carbureto de cálcio ou etefon.

3) A cultivar SNG-2 apresenta maior peso do fruto e menor peso da coroa.

\section{REFERÊNCIAS}

BEZERRA, J. E. F.; LEDERMAN, I. L.; AGUIAR, J. A. E.; REIS, O. V. Influência da idade de indução do florescimento e do peso dos filhotes sobre a produção e qualidade do abacaxizeiro 'Cayenne'. Pesquisa Agropecuária Pernambucana, Recife, v.2, n.1, p.45-55, 1978

CARVALHO, V.D. de. Composição, colheita, embalagem e transporte do fruto. In: CUNHA, G. A. P. da; CABRAL, J. R. S.; SOUZA, L. F. da S. (Ed.) O abacaxizeiro: cultivo, agroindústria e economia. Brasília: Embrapa Comunicação para Transferência de Tecnologia, 1999. p.367388

CHOAIRY, S. A.; FERNANDES, P. D. Épocas de produção de abacaxizeiro no Estado da Paraíba. Pesquisa Agropecuária Brasileira, Brasília, v.19, n.6, p. 729-733, 1984

CHOAIRY, S. A.; FERNANDES, P. D.; OLIVEIRA, E. F. de. Estudos de época de plantio, peso de muda e idade de indução floral em abacaxi cv. Smooth Cayenne. Pesquisa Agropecuária Brasileira, Brasília, v.29, n.1, p. 63-71, 1994a.

CHOAIRY, S. A.; OLIVEIRA, E. F. de; FERNANDES, P. D. Estudos de época de plantio e indução floral em abacaxizeiro Pérola. Pesquisa Agropecuária Brasileira, Brasília, v.29, n.1, p.73-79, 1994b.

CUNHA, G. A. P. da. Florescimento e uso de fitorreguladores. In: CUNHA, G. A. P. da.; CABRAL, J. R. dos S.; SOUZA, L. F. de S. (Ed.) O abacaxizeiro: cultivo, agroindústria e economia. Brasília: Embrapa Comunicação para Transferência de Tecnologia, 1999. p.229-251.
CUNHA, G. A. P. da; REINHARDT, D. H. R. C.; CALDAS, R. C. Efeito da época de plantio, tamanho da muda e idade da planta na indução floral sobre o rendimento do abacaxizeiro 'Pérola' na Bahia. Revista Brasileira de Fruticultura, Cruz das Almas, v.15, n.3, p.43-50, 1993.

GONDIM, T. M. de S.; AZEVEDO, F. F. de. Diferenciação floral do abacaxizeiro cv. SNG-3 em função de idade da planta e da aplicação do carbureto de cálcio. Revista Brasileira de Fruticultura, Jaboticabal, v.24, n.2, p.420-425, 2002.

IBGE. Sistema IBGE de recuperação automática-SIDRA. Disponível em: $<$ http://www.sidra.ibge.gov.br/bda/tabela/protabl.asp $>$. Acesso em: 20 julho 2003

MANICA, I.; FIORAVANÇO, J. C.; BARRADAS, C. I. N.; KIST, H.; VIONE, G. F. Indução do florescimento e produção do abacaxizeiro cv. Smooth Cayenne. Pesquisa Agropecuária Brasileira, Brasília, v.29, n.1, p.81-86, 1994

MELO, G. W. B. de. Época de indução artificial da diferenciação floral do abacaxizeiro 'Pérola' cultivado em área de mata de Roraima. Pesquisa Agropecuária Brasileira, Brasília, v.28, n.10, p.1191-1194, 1993.

PREGNOLATTO, W.; PREGNOLATTO, N. P. Frutas e produtos de frutas. In: PREGNOLATTO, W.; PREGNOLATTO, N. P. (Coord.) Normas analíticas do Instituto Adolfo Lutz: métodos químicos e físicos para análise de alimentos. 3.ed. São Paulo: IAL, 1985. v.1, p.179-188.

REINHARDT, D. H.; SOUZA, L. F. da S.; CABRAL, J. R. S. (Org.) Abacaxi. Produção: aspectos técnicos. Cruz das Almas: Embrapa Mandioca e Fruticultura; Brasília: Embrapa Comunicação para Transferência de Tecnologia, 2000. p.41-44. (Frutas do Brasil, 7).

RITZINGER, R. Avaliação de duas cultivares de abacaxi em Rio Branco, Acre. Revista Brasileira de Fruticultura, Cruz das Almas, v.13, n.3, p.43-48, 1991

RITZINGER, R. Recomendação de cultivares de abacaxi para o Acre. Rio Branco: EMBRAPA-CPAF/AC, 1992. Não paginado.

SAMPAIO, A. C.; CUNHA, R. J. P.; CUNHA, A. R. Influência de nitrogênio e de épocas de plantio sobre o crescimento vegetativo e a diferenciação floral natural do abacaxizeiro cv. Smooth Cayenne. Revista Brasileira de Fruticultura, Cruz das Almas, v.19, n.1, p.714, 1997.

VIEIRA, A.; GADELHA, R. S. de S.; MALDONADO, J. F. M.; SANTOS, A. C. dos. Influência da idade da planta na indução floral sobre a produção do abacaxizeiro. Pesquisa Agropecuária Brasileira, Brasília, v.18, n.1, p.33-35, 1983. 